\title{
Diagnostic, treatment, and reporting criteria for non-specific genital infection in sexually transmitted disease clinics in England and Wales
}

\section{1: Diagnosis}

M. W. ADLER

From the Academic Department of Genitourinary Medicine, Middlesex Hospital Medical School, London

SUMMARY The current methods of diagnosis for non-specific genital infection (NSGI) in clinics in England and Wales are described. In most clinics $(92 \%)$ microscopical findings were used by consultants to establish the diagnosis of non-specific urethritis (NSU) in male patients. However, the microscopical criteria that they used in reaching a diagnosis varied between clinics. The most commonly applied criterion was that of less than five leucocytes per high power field. NSGI in female patients and non-specific proctitis in passive homosexuals were recognised as distinct clinical entitities by only some physicians; the former was acknowledged by consultants working in $60 \%$ of clinics and the latter in $57 \%$. Among those who recognised these conditions the diagnostic criteria varied. The establishment of acceptable and uniform criteria for diagnosis are discussed.

\section{Introduction}

Consultants were first asked to notify non-specific genital infection (NSGI) as a separate item in the quarterly returns in 1971. Previously, since 1951, only cases in men were recorded in the category of 'nongonococcal urethritis'. Since that time the number of cases reported in men has risen by over $500 \%$. Nongonococcal or non-specific urethritis (NSU) is now the most commonly notified sexually transmitted disease (STD) in England and Wales.

Previous papers (Adler et al, 1978; Adler, 1978a, b; Belsey and Adler, 1978) have described the methods used during an inquiry into the facilities and patterns of care for patients with gonorrhoea and herpes genitalis in STD clinics in England and Wales. This paper is concerned with the current management of NSGI by consultants working in 173 clinics for female patients and 171 clinics for male patients. The first part of the paper describes the diagnosis of NSGI and the second the treatment and reporting criteria used in the clinics.

Address for reprints: Professor M. W. Adler, Academic Department of Genitourinary Medicine, James Pringle House, Middlesex Hospital Medical School, London W1N 8AA

Received for publication 16 March 1978

\section{Results}

\section{DIAGNOSTIC CRITERIA}

NSU in male patients

The consultants in charge of clinics were asked to indicate the criteria that they used to make a diagnosis of NSU on microscopical examination of urethral secretions from male patients. Table 1 shows the range of numbers of leucocytes per high power field (HPF) that they used to make a diagnosis, and these varied between clinics. The criteria are not mutually exclusive. The most commonly applied criterion was that of less than five leucocytes per HPF and was used in $113(66 \%)$ clinics. The next

Table 1 Criteria used in clinics to establish diagnosis of NSU by microscopy in male patients

\begin{tabular}{lrr}
\hline & \multicolumn{2}{l}{ Clinics } \\
\cline { 2 - 3 } Microscopical findings & No. & $\%$ \\
\hline Leucocytes/HPF & & \\
$<5$ & 113 & $66 \cdot 1$ \\
$5-9$ & 17 & $9 \cdot 9$ \\
$\geqslant 10$ & 28 & $16 \cdot 4$ \\
Other & 11 & $6 \cdot 4$ \\
Microscopy not used & 2 & $1 \cdot 2$ \\
\hline
\end{tabular}


most frequently applied criterion was that of 10 or more leucocytes per HPF (in $16 \%$ of clinics) followed by 5-9 leucocytes per HPF (in $10 \%$ of clinics). In 11 clinics the criteria used did not involve any attempt to quantify the number of leucocytes. Qualitative criteria, such as clumping, the shape of the white cells, and the absence of Neisseria gonorrhoeae, were applied. In $130(76 \%)$ clinics an early morning urine specimen for microscopical examination was collected if there was any doubt about the diagnosis at the patient's initial visit. In two clinics microscopy was not used, the diagnosis being made in one of them on the basis of the clinical history and two-glass urine test and in the other on purely clinical grounds, without microscopy or urine testing.

In most clinics ( $94 \%$ ) a two-glass urine test was carried out as well as microscopical examination of urethral discharge, and the presence of flakes or threads in the first glass of voided urine was considered to be a positive finding of urethritis. In the absence of positive microscopical results, consultants working in nine clinics were prepared to make the diagnosis of NSU on the evidence of findings in a two-glass urine test in conjunction with the symptom of urethral discharge. In a further eight clinics the diagnosis was made on the basis of a two-glass urine test in the absence of urethral discharge. When the two-glass urine test was used in this way in these 17 clinics no attempt was made to remove or centrifuge the flakes or threads for microscopical examination and the diagnosis was made on the macroscopical evidence alone.

\section{NSGI in female patients}

Physicians working in $103(60 \%)$ clinics for female patients recognised NSGI as a distinct entity even if the patient was not a sexual contact of a man with NSU. Consultants who said that they recognised the condition were asked to specify the criteria that they used in making the diagnosis. The criteria used fell into four categories: (1) microscopical evidence; (2) positive findings on clinical examination; (3) positive clinical history; and (4) positive culture results (Table 2$)$. In $74(72 \%)$ clinics in which the condition was recognised consultants required only one criterion to establish the diagnosis. Physicians using one criterion were not exclusively committed to any particular one. In $22(21 \%)$ clinics two criteria were required and in six $(6 \%)$ three or more.

In clinics in which only one criterion was used to make the diagnosis the most common was that of positive microscopical evidence on its own (52 [50\%] clinics). In clinics in which two criteria were required the most common were positive findings
Table 2 Criteria used to establish diagnosis of NSGI in female patients

\begin{tabular}{|c|c|c|}
\hline & Clinics & $(n=103)$ \\
\hline Criteria & No. & \%o \\
\hline \multicolumn{3}{|l|}{ One criterion $(n=74)$} \\
\hline Positive microscopical findings & 52 & $50 \cdot 5$ \\
\hline Positive clinical findings & 37 & $35 \cdot 9$ \\
\hline Positive clinical history & 22 & $21 \cdot 4$ \\
\hline Positive culture results & 12 & $11 \cdot 7$ \\
\hline \multicolumn{3}{|l|}{ Two criteria } \\
\hline $\begin{array}{l}\text { Positive microscopical and clinical findings } \\
\text { Positive microscopical findings and clinical }\end{array}$ & 11 & $10 \cdot 7$ \\
\hline 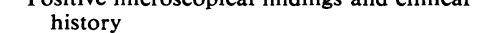 & 10 & $9 \cdot 7$ \\
\hline Positive clinical findings and history & 1 & $1 \cdot 0$ \\
\hline \multicolumn{3}{|l|}{ Three criteria } \\
\hline $\begin{array}{l}\text { Positive microscopical and clinical findings } \\
\text { and clinical history }\end{array}$ & 1 & $1 \cdot 0$ \\
\hline \multicolumn{3}{|l|}{ Four criteria } \\
\hline $\begin{array}{l}\text { Positive microscopical and clinical findings, } \\
\text { history, and culture results }\end{array}$ & 5 & $4 \cdot 9$ \\
\hline Not known & 1 & $1 \cdot 0$ \\
\hline
\end{tabular}

on clinical examination in combination with microscopical evidence or a history of sexual contact plus microscopical evidence.

Positive microscopical evidence was the most frequently used criterion mentioned by consultants regardless of how many they used. Microscopical evidence was one of the criteria in $79(77 \%)$ clinics in which the condition was recognised. However, there was a variation between clinics in the number of leucocytes per HPF that were used. In three clinics less than 10 leucocytes per HPF on the cervical slide constituted a positive diagnosis, in 21 clinics 10 or more, but in most (55) the number of leucocytes was not quantified. The next most frequently used criterion was that of positive findings on clinical examination (55 [53\%] clinics); cervicitis and mucopurulent or purulent cervical discharge were the findings most commonly mentioned. Since there is so much variation in the definition and observation of cervicitis and discharge no attempt was made to define this entity in the study or to ask the physicians to do so. In $17(16 \%)$ clinics positive culture results were mentioned, the most commonly cited organisms being Chlamydia or Ureaplasma urealyticum.

All the consultants who indicated that they recognised the condition of NSGI in women as a distinct clinical entity gave treatment to the patient.

Non-specific proctitis in passive homosexual patients Non-specific proctitis was recognised as a distinct clinical entity by consultants working in $98(57 \%)$ clinics even if the patient was not a sexual contact of a man with NSU. They were asked to stipulate the criteria that they used to reach this diagnosis (Table 3). In most clinics in which the condition 
Table 3 Criteria used to establish diagnosis of non-specific proctitis in passive homosexual patients

\begin{tabular}{|c|c|c|}
\hline & Clinics & $(n=98)$ \\
\hline Criteria & No. & $\%$ \\
\hline \multicolumn{3}{|l|}{ One criterion $(n=75)$} \\
\hline Positive microscopical findings & 61 & $62 \cdot 2$ \\
\hline Positive clinical findings & 23 & $23 \cdot 5$ \\
\hline Positive clinical history & 14 & $14 \cdot 3$ \\
\hline \multicolumn{3}{|l|}{ Two criteria } \\
\hline Positive microscopical and clinical findings & 10 & $10 \cdot 2$ \\
\hline $\begin{array}{l}\text { Positive microscopical findings and clinical } \\
\text { history }\end{array}$ & 6 & $6 \cdot 1$ \\
\hline Positive clinical findings and history & 3 & $3 \cdot 1$ \\
\hline \multicolumn{3}{|l|}{ Three criteria } \\
\hline $\begin{array}{l}\text { Positive microscopical and clinical findings } \\
\text { and clinical history }\end{array}$ & 2 & 2.0 \\
\hline Not known & 2 & 2.0 \\
\hline
\end{tabular}

was recognised consultants required only one criterion to establish the diagnosis; most frequently this was positive microscopical evidence without a supporting positive history or clinical findings on examination ( $62 \%$ of clinics). The next most commonly used criterion was that of positive clinical findings without positive history or microscopical evidence.

In the clinics in which two criteria were used the most common combinations were those of positive findings on clinical examination and microscopical evidence (10 clinics) followed by clinical history and microscopical evidence (six clinics).

Positive microscopical evidence was the most frequently used criterion mentioned by consultants regardless of how many they used. Microscopical evidence was one of the criteria in $79(81 \%)$ clinics in which the condition was recognised: however, the criteria for this varied. In 24 clinics less than 10 leucocytes per HPF on the rectal slide constituted a positive diagnosis and in six clinics 10 or more, but in most clinics (49) the number of leucocytes was not quantified. The next most frequently used criterion was a positive finding on clinical examination such as proctitis; again no attempt was made to define proctitis. No consultants mentioned that they looked for or used any other bacteriological evidence to establish the diagnosis of non-specific proctitis. Naturally, most physicians (in 166 [97\%] clinics) attempted to exclude gonorrhoea by microscopy or culture of rectal specimens or both. Even though all consultants who recognised NSGI in women treated it if the patient fulfilled their criteria, fewer of them ( $93 \%$ ) treated non-specific proctitis.

\section{INVESTIGATIONS BEFORE TREATMENT}

Microscopy

In seven $(4 \%)$ clinics at which male patients were seen and nine $(5 \%)$ at which female patients were seen the results of Gram-stained smears were not available when the patients first attended. Physicians working in the male clinics began treatment in all cases (five clinics) or in selected cases (two clinics) on the basis of the history and clinical findings. Of the nine female clinics without immediate microscopy, patients were treated on the basis of the history and clinical findings in all cases in three clinics and in selected cases in three clinics.

\section{Cultures}

All consultants working in clinics with a culture service for Neisseria gonorrhoeae who had not already begun treatment on the basis of the clinical evidence were prepared to treat male patients on the presumptive results of smears and two-glass urine tests before the culture results were available.

It has been pointed out earlier that physicians working in only $103(60 \%)$ clinics recognised and treated the condition of NSGI in women. In 48 $(47 \%)$ of the clinics in which the condition was treated this was done before the culture results were available. In the remaining clinics the consultants waited for the culture results before giving treatment. They did this in order either to exclude gonorrhoea or to look for other organisms such as Chlamydia and Ureaplasma or both.

\section{EXAMINATION OF SEXUAL CONTACTS \\ Female contacts}

In almost all the clinics $(96 \%)$ at which female patients were seen attempts were made to see all or selected female sexual contacts of male patients with NSU. Physicians working in 76 clinics attempted to see all sexual contacts, but the most frequent practice (in 90 clinics) was to see only selected ones. The two commonest reasons for selection were : (1) that the sexual contact was the patient's regular partner; and (2) that the original male patient had had a recurrence of NSU after an adequate course of treatment (Table 4). In a few clinics sexual contacts were seen if the contact wished to come and attendance at the clinic would not disrupt the relationship or if the patient indicated that the sexual contact had genital symptoms

Table 4 Reasons for seeing selected female contacts of male patients with NSU

\begin{tabular}{lll}
\hline & Clinics & $(n=90)$ \\
\cline { 2 - 3 } Reason & No. & $\%$ \\
\hline Regular sexual partner & 44 & 48.9 \\
Recurrence in original male patient & 40 & $44 \cdot 4$ \\
If convenient & 9 & $10 \cdot 0$ \\
Symptoms/positive history & 7 & $7 \cdot 7$ \\
Others & 9 & $10 \cdot 0$ \\
\hline
\end{tabular}


or a history suggesting infection. In $72(80 \%)$ clinics at which female contacts were selected one of the reasons was specified while in the remaining $20 \%$ of clinics two of the reasons were given.

\section{Homosexual contacts}

Fewer physicians attempted to see passive homosexual contacts of patients with NSU than female contacts. In $120(70 \%)$ clinics attempts were made to see all or selected homosexual contacts. The commonest reasons for selection were: (1) that the contact was a regular partner; (2) that the original patient had had a recurrence; and (3) that the partner was willing to attend.

\section{Discussion}

Even though venereologists have notified NGU, and subsequently NSGI, for only just over 20 years, it has been recognised as a clinical entity-albeit ill defined-for many centuries before this. Boyd (1955) was convinced that a form of non-specific or abacterial urethritis had been described by Galen, Aretaeus, and Celsus in the first and second centuries AD. Harkness (1950) and Csonka (1965) attempted to disentangle the enigma of nongonococcal urethritis and bring some orderliness to this disease or group of diseases. More recently, interest has focused on the role of organisms such as Chlamydia and Ureaplasma urealyticum in the aetiology of this condition (Dunlop et al, 1965; Dunlop et al, 1972; Richmond et al, 1972; Alani et al, 1977; Taylor-Robinson et al, 1977). Unfortunately, facilities for the isolation of these organisms are available in only a few centres. In most cases, however, no specific aetiological factor can be identified, and the diagnosis is made on the clinical history, clinical examination, and exclusion of gonorrhoea, trichomoniasis, and candidosis.

In the absence of gonorrhoea, trichomoniasis, and candidosis microscopical examination of a Gram-stained smear of urethral discharge forms the cornerstone of the diagnosis of NSU, being based on the presence of polymorphonuclear leucocytes. Most authors describing NSU avoid quantification of the leucocytes required to make a positive diagnosis and prefer to use imprecise, qualitative terms such as 'abundant', 'excessive', 'many', or 'significant' etc. Some authors, however, have endeavoured to define what they are describing. Richmond et al (1972) and Alani et al (1977) both suggested that a diagnosis of urethritis could be made in the presence of 10 or more leucocytes per HPF on a urethral smear. Other writers have used more stringent criteria. Prentice (1976) suggested 15 or more leucocytes and Oriel (1976) 20 or more per HPF. Holmes et al (1975) and Bowie et al (1976) defined criteria for centrifuged urine specimens and suggested the presence of 20 leucocytes or more per HPF; Fox (1974) suggested 10 leucocytes per HPF in specimens pepared in the same way.

Even though no formal consensus appears to exist in England and Wales on the appropriate quantification of leucocytes it has been shown in the present survey that informal agreement does exist. In most clinics $(66 \%)$ the criteria of less than five leucocytes per HPF was used whereas in $26 \%$ of clinics five or more leucocytes per HPF were required, and in a further $8 \%$ no attempt was made at quantification.

Some venereologists will submit that a leucocyte count of less than five per HPF is too low, whereas others will argue that a patient with urethritis due to Chlamydia can have both a minimal count and a urethral discharge which would be left undiagnosed and untreated if higher counts were required. While there is little to be gained from argument about whether the use of less than five leucocytes per HPF is too low it must be realised that one physician's definition of NSU is not necessarily another's. It is extremely unsatisfactory that when NSU is discussed, described, diagnosed, and notified no accepted criteria are in existence. The ideal solution would be for all physicians to agree on a standard criterion and universally applied 'cut off' point for establishing the diagnosis if no organism is isolated. Such a step would remove some of the imprecision surrounding the diagnosis and definition of this disease. It must be realised that if those working in the field of genitourinary medicine decided to recommend that the diagnosis of NSU be made on the basis of less than five leucocytes per HPF the number of reported cases will appear to increase, since physicians using the more stringent criteria will start to diagnose and report patients as having NSU who would have previously been considered normal. If a standard criterion is not acceptable to venereologists it might be possible to persuade them to indicate their own individual criterion currently in use to establish a diagnosis.

Physicians working in 17 clinics made a diagnosis of NSU on the basis of the two-glass urine test in the absence of symptoms. The two-glass urine test is subjective, unreliable, and no substitute for microscopy. The difficulties in establishing universally acceptable microscopical criteria should not deter doctors from using this method as the basis for diagnosis as opposed to relying on the two-glass urine test alone.

Despite the imperfections of making the diagnosis of NSU in male patients, all the venereologists 
acknowledged that the disease existed. This was not so for NSGI in women. Consultants working in only $60 \%$ of clinics recognised the condition as a distinct clinical entity even though the patient was not a sexual contact. The two most commonly used criteria in clinics in which the condition was recognised were those of positive microscopical findings ( $77 \%$ of clinics) and positive findings on clinical examination such as cervicitis or mucopurulent or purulent cervical discharge or both (53\% of clinics).

Attempts have been made to establish criteria for the diagnosis of NSGI in women. Fox (1974) suggested 50 or more leucocytes in several HPFs on a cervical smear in conjunction with inflammatory changes on cervical cytology smears. Other workers have used cervical cytology to make the diagnosis. Simmons and Vosmik (1974) graded the level of inflammation in female sexual contacts of men with NSU and suggested that the presence of inflammatory changes on cytology might help in the diagnosis of NSGI. These changes have also been reported by Burns et al (1975). Atia and Thin (1975) also looked at the value of cytology in diagnosing genital infection and showed that women with gonorrhoea, trichomoniasis, and-to a lesser extent -candidosis exhibited inflammatory changes and that it was important to exclude these diseases before the patient was diagnosed as having NSGI. Earle (1977) came to the same conclusion when he examined urethral specimens for leucocytes.

There is a clear paradox in relation to the presence of leucocytes in the female genital tract. Most venereologists and gynaecologists consider that, on the one hand, leucocytes occur in association with infection by a specific organism and that, on the other, in the absence of gonorrhoea, trichomoniasis, candidosis, chlamydial or other infections they are of little importance since they are an expected and normal finding in the genital tract. 'In the absence of a contact history there is no certain way of identifying women who may require treatment for non-specific genital infection. In particular the presence of clinical signs of cervical inflammation, or inflammatory changes on cervical cytology, are not necessarily diagnostic and are not in themselves a certain indication for antibiotic therapy' (Oriel, 1976). A further problem arises when a decision has to be made whether to treat all sexual contacts or only those with evidence of infection, such as cervicitis or mucopurulent or purulent cervical discharge. Csonka (1972) has advocated treatment only in the presence of cervicitis or pelvic infection. In the absence of any clear guide lines as to what the signs and microscopical findings of this condition are, it might be preferable to treat all sexual con- tacts epidemiologically on the basis of a history of sexual contact or selectively, as in instances such as reinfection of the male contact. In the present survey physicians working in approximately half the clinics treated all sexual contacts regardless of whether or not evidence of infection was present. In the remaining $50 \%$ of clinics patients were treated only in selected instances; none of the consultants mentioned the presence of cervicitis or purulent cervical discharge as a reason for this selection. This would appear to be a reasonable and pragmatic approach to an ill-defined and ill-understood problem.

Oriel's (1976) statement with regard to NSGI as a distinct entity highlights the dilemma surrounding this condition in women. Even though a substantial number of physicians recognised this as a distinct entity, does it really exist as such? Those who believe in its existence should be encouraged and challenged to suggest acceptable and reproducible criteria for establishing the diagnosis. Until this is done, would it not be better to accept that the disease does not exist as a primary entity in women?

Fewer consultants recognised non-specific proctitis as a distinct clinical entity than NSGI in female patients. In both instances there is little agreement as to whether or not the condition exists and, if it does, as to the appropriate criteria for diagnosis. Fluker (1976) has summed this up: 'the criteria for diagnosis are as nebulous as those for non-specific genital infection in the female and largely depend on known contact with a case of non-specific urethritis in the absence of Neisseria gonorrhoeae in smears and cultures'. Once again, it is obvious that work is required to establish more accurate and acceptable criteria, and that at present it is preferable to accept that non-specific proctitis is not a distinct entity.

\section{Conclusions}

Non-specific genital infection is now the commonest diagnosis made in STD clinics in England and Wales yet the one with the least uniformity in the establishment of a diagnosis and the notification of cases. The absence of a causative organism which can be identified by routine laboratory methods in most cases makes it all the more important that a standard approach to diagnosis is developed. This may not be possible in women. Work with Chlamydia trachomatis has shown that a diagnosis of chlamydial cervical infection cannot be made by clinical examination, microscopy, and exclusion of Neisseria gonorrhoeae and Trichomonas vaginalis. 


\section{References}

Adler, M. W., Belsey, E. M., O’Connor, B. H., Catterall, R. D., and Miller, D. J. (1978). Facilities and diagnostic criteria in sexually transmitted disease clinics in England and Wales. British Journal of Venereal Diseases, 54, 2-9.

Adler, M. W., (1978a). Diagnostic, treatment and reporting criteria for gonorrhoea in sexually transmitted disease clinics in England and Wales: 1 Diagnosis. British Journal of Venereal Diseases, 54, 10-14.

Adler, M. W. (1978b). Diagnostic, treatment and reporting criteria for gonorrhoea in sexually transmitted disease clinics in England and Wales: 2 Treatment and reporting criteria. British Journal of Venereal Diseases, 54, 15-23.

Alani, M. D., Darougar, S., Burns, D. C. MacD., Thin, R. N., and Dunn, H. (1977). Isolation of Chlamydia trachomatis from the male urethra. British Journal of Venereal Diseases, 53, 88-92.

Atia, W. A., and Thin, R. N. (1975). Cervical cytology in genital infection. British Journal of Venereal Diseases, 51, 331-332.

Belsey, E. M., and Adler, M. W. (1978). Current approaches to the diagnosis of herpes genitalis. British Journal of Venereal Diseases, 54, 115-120.

Bowie, W. R., Floyd, J. F., Miller, Y., Alexander, F. R., Holmes, J., and Holmes, K. K. (1976). Differential response of chlamydial and ureaplasma-associated urethritis to sulphafurazole (sulfisoxazole) and aminocyclitols. Lancet, 2, 1276-1278.

Boyd, R. H. (1955). Origin of gonorrhoea and non-specific urethritis. British Journal of Venereal Diseases, 31, 246-248.

Burns, D. C. MacD., Darougar, S., Thin, R. N., Lothian, L., and Nicol, C. S. (1975). Isolation of Chlamydia from women attending a clinic for sexually transmitted disease. British Journal of Venereal Diseases, 51, 314-318.

Csonka, G. W. (1965). Non-gonococcal urethritis. British Journal of Venereal Diseases, 41, 1-8.

Csonka, G. W. (1972). Venereal diseases. Medicine, 6, 502-513.

Dunlop, E. M. C., Al-Hussaini, M. K., Garland, J. A., Treharne, J. D., Harper, I. A., and Jones, B. R. (1965). Infection of urethra by TRIC agent in men presenting because of 'non-specific urethritis', Lancet, 1, 1125-1128.
Dunlop, E. M. C., Vaughan-Jackson, J. D., Darougar, S., and Jones, B. R. (1972). Chlamydial infection: incidence in 'nonspecific' urethritis. British Journal of Venereal Diseases, 48, 425-428.

Earle, J. V. (1977). Significance of leucocytes in smears taken from the female urethral meatus. British Journal of Venereal Diseases, 53, 56-57.

Fluker, J. L. (1976). A 10-year study of homosexually transmitted infection. British Journal of Venereal Diseases, 52, 155-160.

Fox, H. (1974). Non-specific genital infection in a general practice. British Journal of Venereal Diseases, 50, 125-131.

Harkness, A. H. (1950). Non-gonococcal urethritis. Livingstone: Edinburgh.

Holmes, K. K., Handsfield, H. H., Wang, S. P., Wentworth, B. B. Turck, M., Anderson, J. B., and Alexander, F. R. (1975). Aetiology of non-gonococcal urethritis. New England Journal of Medicine, 292, 1199-12.

Oriel, J. D. (1976). Non-specific urethritis and vaginal discharge. Prescribers' Journal. 16, 108-116.

Prentice, M. J., Taylor-Robinson, D., and Csonka, G. W. (1976) Non-specific urethritis. A placebo controlled trial of minocycline in conjunction with laboratory investigations. British Journal of Venereal Diseases, 52, 269-275.

Richmond, S. J., Hilton, A. L., and Clarke, S. K. R. (1972). Chlamydial infection: role of Chlamydia subgroup $\mathbf{A}$ in non-gonococcal and post-gonococcal urethritis. British Journal of Venereal Diseases, 48, 437-444.

Simmons, P. D., and Vosmik, F. (1974). Cervical cytology in nonspecific genital infection: an aid to diagnosis. British Journal of Venereal Diseases, 50, 313-314.

Taylor-Robinson, D., Csonka, G. W., and Prentice, M. S. (1977). Human intra-urethral inoculation of ureaplasmas. Quarterly Journal of Medicine, 46, 309-326. 